\title{
Crisp Function of Integral Nonlinaer Equation of the Second Kind over the Fuzzy Interval with Application
}

\author{
Alan Jalal Abdulqader \\ Department of Mathematics and Natural Science, Faculty MIPA, University Gadjah Mada, Yogyakarta, Indonesia \\ Email address: \\ alanjala1515@yahoo.com (A. J. Abdulqader) \\ To cite this article: \\ Alan Jalal Abdulqader. Crisp Function of Integral Nonlinaer Equation of the Second Kind over the Fuzzy Interval with Application. \\ American Journal of Applied Mathematics. Vol. 3, No. 4, 2015, pp. 189-200. doi: 10.11648/j.ajam.20150304.15
}

\begin{abstract}
In this paper, the basic principle and definitions for nonlinear integral equation of a crisp function over a fuzzy interval have been discussed. a numerical technique method and some algorithm for solving non-linear of crisp valued function over fuzzy interval using the domain and range partitions of the membership functions of the fuzzy interval . the numerical solution of the crisp function over the fuzzy interval using the LR-type representation of fuzzy interval. Some numerical examples are prepared to show the efficiency and accuracy of the methods.
\end{abstract}

Keywords: Fuzzy Number, Volterra Non-linear Integral Equation of Second Kind, Trapezoidal Quadrature Method, Fuzzy Interval, LR-type of Fuzzy Interval

\section{Introduction}

The basic sciences ( such as engineering, chemistry, and physics) construct exact mathematical models of empirical phenomena, and then using these models for making predictions, while some aspects of real world problems always escape from such precise mathematical models, an usually there is one elusive inexactness as a part of the original model[kandel, 1986]. Scientists have long sought ways to use the precision of mathematics to tame the imprecision of the real world. It may be seen that in manyvalued logic, topology, and probability theory as different attempts to be precise about imprecision [Negoite, 1975], [Zadeh, 1965] published his classical paper "fuzzy set theory " which is received more and more attention from researcher in wide rang of scientific areas especially in the past few years[Dubois, 1980].

Nowadays, they are equipped with their owe mathematical foundation, rooting from set- theoretic basis and manyvalued logic. Their achievements have already enriched the classic two-valued logic with a deep and novel perspective and to understand the reasons for the extensive development of fuzzy sets, there are two main aspects worthily of being mentioned. Firstly, the notion of fuzzy set as a too; for modeling intermediate grades of belonging that occur in any concept, is very attractive, especially from an application point of view. Secondly a variety of tools incorporated in the framework of fuzzy sets enables to find a suitable concept to cope with the theory of fuzzy sets has one of it's aims the development of a methodology for the formulation and solution of problems that are too complex or too ill-defined to be susceptible to analysis by conventional techniques [Kandel, 1986]. The basic idea of fuzzy sets is easy to grasp, let us remind ourselves of two- valued logic, which forms a corner stone of any mathematical tool used. A fundamental point arising from this logic is that it imposes a dichotomy of any mathematical model, in other words taking any object, we are forced to assign it to one of two prespecified categories (for example, good - bad , black- white , normal abnormal, odd- even, etc).

Sometime it happened that this process of classification may easily performed, since the categories we are working with are precise and well-defined, for instance, with two categories of natural number as belonging to exactly one class.

Nevertheless, in many scientific tasks, we faced with classes that are ill-defined. Consider for instance, such as tall man, high speed, significant error, etc. All of these convey a useful semantic meaning that is obvious for a certain community, however, a borderline between the belonging or not of a given object to such a class is not evident. Here, it is obvious that two-valued logic, used in describing these classes of situations, might be not well suited. A historical example appeared in one of the work (Borel, who discussed an ancient Greek sophism of the pile of seeds ... one seed 
dose not constitute a pile nor two three. From the other side everybody will agree that 100 million seeds constitute a pile. What therefor is the appropriate limit? Can we say that 325647 seeds don't constitute a pile but 325648 do?). Therefore, even in mathematics we can meet some fuzzy notations (example ill-conditioned matrix, sparse matrix).

Techniques of fuzzy sets and systems theory were applied in various domains such as: pattern recognition, decisionmaking under uncertainty, large- system control, management science, and others [Dubois, 1980].

One of the most important facts of human thinking is the ability to summarize information into label of fuzzy sets, which bear an approximate relation to the primary data [Dubois , 1980].

The fuzziness is a type of imprecision that stems from grouping of element into classes that do not have defined boundaries, such classes called fuzzy set, arises whenever we describe the ambiguity, vagueness and ambivalence in the mathematical model of empirical phenomena [Kandel, 1986].

In general we distinguish three kinds of exactness. Generality that a concept applies to a variety of situations. Ambiguity that it describes more than one distinguishable sub- concept, vagueness, that precise boundaries are not defined. All three types of inexactness are represented by fussy set. Generality occurs when the universe is not just one point, ambiguity occurs when there is more than one local maximum of membership function, and vagueness occurs when the function take value other than just (0and 1) [kandel, 1986].

In this paper, we construct a new technique to find a solution of the NON-linear integral equation of a crisp function over a -fuzzy interval.

$$
u(x)=f(x)+\lambda \int_{\tilde{a}}^{\tilde{b}} k(x, t, u(t)) d t
$$

\section{Fuzzy Sets Theory}

Fuzzy set theory is generalization of abstract set theory; it has a wider scope of applicability than abstract set theory in solving problems tha involve to some degree subjective evaluation [12,19,22].

Let $\mathrm{X}$ be a space of object and $\mathrm{x}$ be a generic element of $\mathrm{X}$, a classical set $\mathrm{A}, \mathrm{A} \subseteq X$ is defined as a collection of elements or objects $\mathrm{x} \in X$, such that each element $\mathrm{x}$ can either belong or not to the set A. By defining a characteristic (or membership) function for each element $\mathrm{x}$ in $\mathrm{X}$, we can represent a classical set $\mathrm{A}$ by a set of ordered pairs $(\mathrm{x}, 0)$ or (x,1), which indicates $x \notin A$ or $x \in A$, respectively. A fuzzy set express the degree to which an element belongs to a set . Hence, for simplicity, the membership function of a fuzzy set is allowed to have value between ( 0 and 1$)$ which denotes the degree of membership of an element in the given set

$\mu_{\tilde{A}}: X \rightarrow[0,1]$, the fuzzy set $\tilde{A}$ in $X$ is defined as a set of ordered pairs

$\tilde{A}=\left\{\left(x, \mu_{\tilde{A}}(x), x \in X\right\} \quad\right.$ where $\mu_{\tilde{A}}(x)$ is called the membership function (or MF ) for the fuzzy set [Bezdek, 1993]
Remarks 1

1- When $\mathrm{X}$ is finite set, a fuzzy set on $\mathrm{X}$ is expressed as:

$$
\begin{aligned}
\tilde{A}=\mu_{\tilde{A}}\left(x_{1}\right) \mid x_{1}+ & \mu_{\tilde{A}}\left(x_{2}\right)\left|x_{2}+\cdots \ldots+\mu_{\tilde{A}}\left(x_{n}\right)\right| x_{n} \\
& =\sum_{i=1}^{n} \mu_{\tilde{A}}\left(x_{i}\right) \mid x_{i}
\end{aligned}
$$

when $\mathrm{X}$ is not finite, we have

$$
\tilde{A}=\mu_{\tilde{A}}\left(x_{1}\right)\left|x_{1}+\mu_{\tilde{A}}\left(x_{2}\right)\right| x_{2}+\cdots \ldots \ldots .=\int_{x} \mu_{\tilde{A}}(x) \mid x
$$

$\mathrm{Or}$

$$
\tilde{A}=\left\{\left(x, \mu_{\tilde{A}}(x), x \in X\right\}\right.
$$

Where the $\operatorname{slash}(\mid)$ is employed link the elements of the support with their grades of membership in $\tilde{A}$, and the plus sign $(+)$ or the integral playing the role of "union" rather than arithmetic sum or integral $[14,17]$.

2 - The biggest differences between crisp and fuzzy set is that the former always have unique memberships, where as every fuzzy set has infinite number of memberships that may represented it .

3- Function that map $X$ into the unit interval may be fuzzy sets but become fuzzy set when, and only when, they match some intuitively plausible semantic description of imprecise properties of the objects in $\mathrm{X}$

\section{Basic Concepts}

Let $\mathrm{X}$ be a space of object, let $\tilde{A}$ be a fuzzy set in $\mathrm{X}$ then one can define the following concepts related to fuzzy subset $\tilde{A}$ of $\mathrm{X}[1,2,22]$ :

1- The support of $\tilde{A}$ in the universal $\mathrm{X}$ is crisp set , denoted by :

$$
S(\tilde{A})=\left\{x \mid \mu_{\tilde{A}}(x)>0, \text { for all } x \in X\right\} .
$$

2- The core of a fuzzy set $\tilde{A}$ is the set of all point $x \in X$, $\operatorname{such}$ that $\mu_{\tilde{A}}(x)=1$

3- The height of a fuzzy set $\tilde{A}$ is the largest membership grade over $\mathrm{X}$, i.e $\operatorname{hgt}(\tilde{A})=\sup _{x \in X} \mu_{\tilde{A}}(x)$

4- Crossover point of a fuzzy set $\tilde{A}$ is the point in $\mathrm{X}$ whose grade of membership in $\tilde{A}$ is 0.5

5- Fuzzy singleton is a fuzzy set whose support is single point in $\mathrm{X}$ with $\mu_{\tilde{A}}(x)=1$

6- A fuzzy set $\tilde{A}$ is called normalized if it is height is 1 ; otherwise it is subnormal

7- The empty set $\phi$ and $X$ are fuzzy set, then : for all $x \in X, \mu_{\phi}(x)=0, \mu_{x}(x)=1$ respectively

8- $\quad \tilde{A}=\tilde{B}$ if and only if $\mu_{\tilde{A}}(x)=\mu_{\tilde{B}}(x)$ for all $\mathrm{x} \in X$

9- $\tilde{A} \subseteq \tilde{B}$ if and only if $\mu_{\tilde{A}}(x) \leq \mu_{\tilde{B}}(x)$ for all $\mathrm{x} \in X$

10- $\tilde{A}^{c}$ is a fuzzy set whose membership function is defined by : $\mu_{\tilde{A}^{c}}(x)=1-\mu_{\tilde{A}}(x)$ for all $x \in X$

11- Given two fuzzy sets, $\tilde{A}$ and $\tilde{B}$, their standard intersection , $\widetilde{A} \sqcap \widetilde{B}$, and the standard union $\tilde{A} \sqcup \widetilde{B}$, are fuzzy sets and their membership function are defined for simplicity for all 
$x \in X$, by the equations:

$$
\begin{aligned}
& \forall x \in X, \mu_{A \cup B}(x)=\operatorname{Max}\left[\mu_{A}(x), \mu_{B}(x)\right] \\
& \forall x \in X, \mu_{A \cap B}(x)=\operatorname{MIn}\left[\mu_{A}(x), \mu_{B}(x)\right]
\end{aligned}
$$

\section{4. $\alpha$-Cut Sets}

One of the most important concepts of fuzzy sets is the concept of an $\alpha$-cut and it is variant, a strong alpha- cut, given a fuzzy set $\tilde{A}$ defined on $\mathrm{X}$ and any number $\alpha \in[0,1]$, the $\alpha$-cut, $A_{\alpha}$ (the strong $\alpha$-cut , $A_{\alpha+}$ ) is the crisp set that contains all elements of the universal set $X$ whose membership grades in $\tilde{A}$ are greater then or equal to (only greater than ) the specified value of $\alpha[3,7]$.

$$
\begin{gathered}
A_{\alpha}=\left\{x \in X: \mu_{A}(x) \geq \alpha\right\}, \forall x \in X \\
A_{\alpha+}=\left\{x \in X: \mu_{A}(x)>\alpha\right\}, \forall x \in X
\end{gathered}
$$

The following properties are satisfied for all $\alpha \in[0,1]$

i $(A \cup B)_{\alpha}=A_{\alpha} \cup B_{\alpha}$

ii $(A \cap B)_{\alpha}=A_{\alpha} \cap B_{\alpha}$

iii $\tilde{A} \subseteq \tilde{B}$ gives $\tilde{A}_{\alpha} \subseteq \widetilde{B}_{\alpha}$

iv $\tilde{A}=\tilde{B}$ iff $A_{\alpha}=B_{\alpha}, \forall \alpha \in[0,1]$

$\mathrm{v} \alpha \leq \alpha^{\prime} \in[0,1]$, If $\alpha \leq \alpha^{\prime}$ then $A_{\alpha} \supseteq A_{\alpha^{\prime}}$

Remarks 2 [11]:

1 - The set of all level $\alpha \in[0,1]$, that represent distinct $\alpha-$ cuts of a given fuzzy set

\section{$\tilde{A}$ is called a level set of $\tilde{A}$}

$$
A(\tilde{A})=\left\{\alpha \mid \mu_{\tilde{A}}(x)=\alpha, \text { for some } x \in X\right\}
$$

2- The support of $\tilde{A}$ is exactly the same as the strong $\alpha-$ cut of $\tilde{A}$ for $\alpha=0, A_{0+}=S(\tilde{A})$.

3 - The core of $\tilde{A}$ is exactly the same as the $\alpha$-cut of $\tilde{A}$ for $\alpha=1$, (i.e $\left.A_{1}=\operatorname{core}(\tilde{A})\right)$.

4- The height of $\tilde{A}$ may also be viewed as the supremum of $\alpha-$ cut for which $A_{\alpha} \neq \phi$

5- The membership function of a fuzzy set $\tilde{A}$ can be expressed in terms of the characteristic function of it is $\alpha-$ cuts according to the formula:

$$
\mu_{\tilde{A}}(x)=\sup _{\alpha \in] 0,1]} \operatorname{Min}\left\{\alpha, \mu_{A^{\alpha}}(x)\right\}
$$

Where

$$
\mu_{A^{\alpha}}(x)=\left\{\begin{array}{c}
1 \text { if } x \in A_{\alpha} \\
0, \text { otherwise }
\end{array}\right.
$$

Definition 1 :

A fuzzy set $\tilde{A}$ on $\mathrm{R}$ is convex if and only if $[5,11]$

$\operatorname{Min}\left\{\mu_{\tilde{A}}\left(x_{1}\right), \mu_{\tilde{A}}\left(x_{2}\right)\right\} \quad$ (2), for all $x_{1}, x_{2} \in R$, and all $\lambda \in$ $[0,1]$

Remarks 3 [12]:

$\mathrm{i}$ Assume that $\tilde{A}$ is convex for all $\alpha$ and let $\alpha=$ $\mu_{\tilde{A}}\left(x_{1}\right), \mu_{\tilde{A}}\left(x_{2}\right)$ then if $x_{1}, x_{2} \in A_{\alpha}$ and moreover $\lambda x_{1}+(1-\lambda) x_{2} \in A_{\alpha}$ for any $\lambda \in[0,1]$ by the convexity of $\tilde{A}$. Consequently $\mu_{\tilde{A}}\left(\lambda x_{1}+(1-\lambda) x_{2}\right) \geq$ $\alpha=\mu_{\tilde{A}}\left(x_{1}\right)=\operatorname{Min}\left\{\mu_{\tilde{A}}\left(x_{1}\right), \mu_{\tilde{A}}\left(x_{2}\right)\right\}$.

ii Assume that $\tilde{A}$ satisfies equation (2), we need to prove that

For any $\alpha \in[0,1], A \_\alpha$ is convex .Now for any $x \_1, x \_2$ $\in$ A_ $\alpha$ and for any $\lambda \in[0,1]$ by equation (2)

$$
\begin{gathered}
\mu_{\tilde{A}}\left(\lambda x_{1}+(1-\lambda) x_{2}\right) \geq \operatorname{Min}\left\{\mu_{\tilde{A}}\left(x_{1}\right), \mu_{\tilde{A}}\left(x_{2}\right)\right\} \\
\geq \operatorname{Min}\{\alpha, \alpha\}=\alpha
\end{gathered}
$$

i.e

$\lambda x_{1}+(1-\lambda) x_{2} \in$

$A_{\alpha}$, therefore $A_{\alpha}$ is convex for any $\alpha \in[0,1], \tilde{A}$ is convex.

\section{Fuzzy Integration of a Crisp (Real- Valued) Function over a Fuzzy Interval [Dubois, 1982a]}

We shall consider a case for which Dubois and Prade $[8,20,21]$ have proposed a fuzzy domain $\mathrm{D}$ of the real line $\mathrm{R}$ assumed to be delimited by two bounds $\tilde{A}$ and $\tilde{B}$ in the following sense

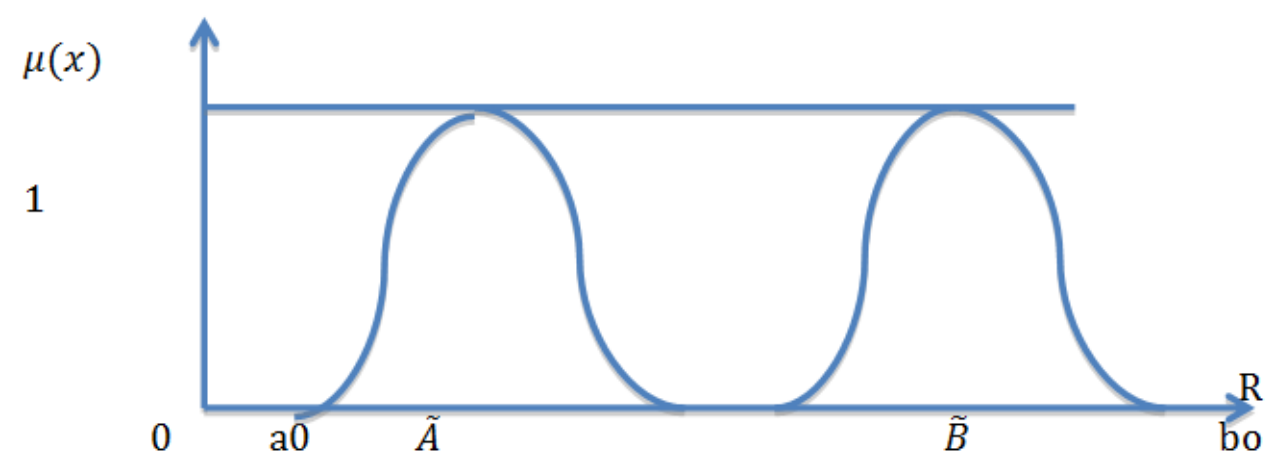

Fig. 1. crisp valued function over a fuzzy interval.

1- $\tilde{A}$ and $\tilde{B}$ are fuzzy sets on $\mathrm{R}$, whose membership function are $\mu_{\tilde{A}}$ and $\mu_{\tilde{B}}$ repectively, from $\mathrm{R}$ to $[0,1]$.
2- $\forall x \in R, \mu_{\tilde{A}}(x)\left(\right.$ respectively $\left.\mu_{\tilde{B}}(x)\right)$ evaluates to what extent $\mathrm{x}$ can be considered as a greatest lower 
bound ( respectively least upper bound ) of $\mathrm{D}$

3- $\tilde{A}$ and $\tilde{B}$ are normalized fuzzy sets.

4- $\tilde{A}$ and $\tilde{B}$ are convex fuzzy set

$D$ is denoed $(\tilde{A}, \tilde{B}), \tilde{A}$ and $\widetilde{B}$ are assumed ordered in the sense that

$$
a_{0}=\operatorname{Inf} S(\tilde{A}) \leq \operatorname{Sup} S(\tilde{B})=b_{0}
$$

where $S($.$) stands for support$

Definition 2 :Let $f(u)$ be a real- valued mapping, and integerable on the interval $1=[\operatorname{Inf} S(\tilde{A}), \operatorname{Sup} S(\tilde{B})]$, then the integral of $\mathrm{f}(\mathrm{u})$ over the domain delimited is defined according to the extension principle by:

$$
\mu_{I(\tilde{x}, \tilde{y})}(Z)=\operatorname{Sup}_{x, y \in I: z=\int_{x}^{y} f(u) d u} \min \left\{\mu_{\tilde{A}}(x), \mu_{\tilde{B}}(y)\right\}
$$

To develop an applicable numerical algorithm for computing fuzzy integration, it is very important to discuss the following useful remarks and propositions.

Remarks 5:

1 - If one of he bounds is not fuzzy, we consider the integral of $f(u)$ over $[a, \tilde{B})$ as $I(a, \tilde{B})$ and it's membership function can be defined as [Dubois , 1980]

$$
\begin{gathered}
\mu_{I(a, \tilde{B})}(z)=\operatorname{Sup}_{y: z=\int_{a}^{y} f(u) d u} \mu_{B}(y) \\
=\operatorname{Sup}_{y: F(y)-F(a)} \mu_{B}(y)
\end{gathered}
$$

where $\mathrm{F}$ is an anti-derivative of $\mathrm{f}$

2- If both bounds are fuzzy, then (2.1) can be rewritten as:

$$
\begin{gathered}
\mu_{I(\tilde{A}, \tilde{B})}(z)=\operatorname{Sup}_{z=F(y)-F(x)} \min \left\{\mu_{\tilde{A}}(x), \mu_{\tilde{B}}(y)\right\} \\
=\operatorname{Sup}_{x \in R} \min \left\{\mu_{\tilde{A}}(x), \operatorname{Sup}_{y: z=F(y)-F(x)} \mu_{\tilde{B}}(y)\right\} \\
=\operatorname{Sup}_{x \in R} \min \left\{\mu_{\tilde{A}}(x), \mu_{I(a, \tilde{B})}(Z)\right\}
\end{gathered}
$$

The following are some of the useful properties:

Proposition 1.[10,]

Let $F(x)$ be an anti-derivative function of $\mathrm{f}(\mathrm{x})$, i.e, $F(x)=\int_{c}^{x} f(x) d x$, for some $c \in I$ ( the interval of integration) denoted $F(\tilde{A})$ is the image of the fuzzy set $\tilde{A}$ through $F$, defined by the extension principle $\forall z \in R$,

$$
\mu_{F(\widetilde{A})}(Z)=\operatorname{Sup}_{x: Z=F(x)} \mu_{\widetilde{A}(x)} .
$$

Moreover , $\ominus$ denotes extended principle the following proposition holds:

$$
\int_{\tilde{A}}^{\tilde{B}} f=F(\tilde{B}) \ominus f(\tilde{A})
$$

Proposition 2. [10,4]

Let $f$ and $g$ be two real mapping integerable on interval I, $(f: I \rightarrow R, g: I \rightarrow R)$ then :

$$
\int_{\tilde{A}}^{\tilde{B}}(f+g) \subseteq \int_{\tilde{A}}^{\tilde{B}} f \oplus \int_{\tilde{A}}^{\tilde{B}} g
$$

where $\subseteq$ denotes the usual fuzzy set inclusion, $\bigoplus$ denotes the extended addition proposition 3.[10,6]

If $f$ and $g$ are both either positive or negative integerable real mapping

$\left(f: I \rightarrow R^{+}, g: I \rightarrow R^{+}\right)$or $\left(f: I \rightarrow R^{-}, g: I \rightarrow R^{-}\right), \quad$ then the equality holds, i.e

$$
\int_{\tilde{A}}^{\tilde{B}}(f+g)=\int_{\tilde{A}}^{\tilde{B}} f \oplus \int_{\tilde{A}}^{\tilde{B}} g
$$

Proposition 4.[10,15]

Let $\dot{D}$ and $\ddot{D}$ be domains of $\mathrm{R}$ delimited by fuzzy bounds $(\tilde{A}, \tilde{C})$ and $(\tilde{C}, \tilde{B})$ respectively, then for any integerable mapping

$$
\int_{D} f \subseteq \int_{\dot{D}} f \oplus \int_{\ddot{D}} f
$$

where $D$ is delimited by $(\tilde{A}, \tilde{B})$, the equality holds if and only if $\tilde{C}$ is real number

Proof:

$$
\begin{gathered}
\int_{D} f=F(\tilde{B}) \ominus F(\tilde{A}) \\
\int_{\dot{D}} f \oplus \int_{\ddot{D}} f=(F(\tilde{B}) \ominus F(\tilde{C})) \oplus(F(\tilde{C}) \ominus F(\tilde{A}))
\end{gathered}
$$

Not that $F(\tilde{C})) \ominus(F(\tilde{C})=0$ if and only if $\tilde{C}$ is real number, otherwise

$V(z)$

$\left.=\operatorname{Sup}_{z=u-v+w-t} \min \left\{\mu_{F(\widetilde{B})}(u), \mu_{F \widetilde{(C)}}(v), \mu_{F(\tilde{c})}(w), \mu_{F(\tilde{A})}(t)\right\}\right\}$

$$
\geq \operatorname{Sup}_{z=u-t} \min \left(\mu_{F(\widetilde{B})}(u), \mu_{F(\widetilde{A})}(t)\right)=\mu(z)
$$

Since we add the constraint $v=w$, and we can $\operatorname{drop} F(\tilde{C})$ which is normalized

Remarks 5.[10,16]

$1-I(a, \tilde{B})=F(\tilde{B}) \ominus F(a)$ is the value of the extended $F(x)-F(a)$, when $x=\tilde{B}$

Proof :

Let $\mu$ be the membership function of $I(a, \tilde{B})$ and $v$ be the membership function

$$
F(\tilde{B}) \ominus F(a)
$$

$$
\begin{aligned}
& V(z) \\
& =\operatorname{Sup}_{u, v: z=u-v} \min \left\{\operatorname{Sup}_{a: u=F(a)} \mu_{a}(a), \operatorname{Sup}_{y: v=F(y)} \mu_{\tilde{B}}(y)\right\} \\
& =\operatorname{Sup}_{u, v: z=u-v} \min \left\{\mu_{a}(a), \operatorname{Sup}_{y: v=F(y)} \mu_{\tilde{B}}(y)\right\} \\
& \quad=\operatorname{Sup}_{a, y: F(y)-F(a)=z} \min \left\{\mu_{a}(a), \mu_{\tilde{B}}(y)\right\}
\end{aligned}
$$

where

$$
F(y)-F(a)=\int_{a}^{y} f(x) d x
$$

$2-I(\tilde{A}, \tilde{B})$ is the fuzzy value of the extended fuzzifying function :

$$
y=F(B) \ominus F(x), \text { for } X=\tilde{A}
$$




\section{Numerical Fuzzy Integration of a Crisp Function over a Fuzzy Interval}

We shall develop a computational algorithm for computing a numerical fuzzy integration of crisp function over a fuzzy interval. The basic principle of this technique for continuous fuzzy number. The continuous fuzzy number is discretized and then converted into discrete fuzzy number so that the numerical fuzzy integration over the discrete case can be easily implemented .

Mathematically, we can represent the fuzzy integration:

$$
I(\tilde{A}, \tilde{B})=\left\{\int_{\tilde{A}}^{\tilde{B}} f(u) d u \mid \tilde{A} \text { and } \tilde{B} \text { are fuzzy numbers and } f(u)\right. \text { is integrable }
$$

valued real function from $\mathrm{R}$ to $\mathrm{R}$ \}

For discrete fuzzy numbers, we develop the following

\subsection{Fuzzy Integration for Discrete Fuzzy Numbers}

$A$ general procedure for computing the fuzzy integration for discrete fuzzy numbers $\widetilde{A}$ and $\tilde{B}$ have been developed and as follows:

Let the universe set be $X$, when $\tilde{A}$ and $\tilde{B}$ are discrete fuzzy numbers,

Then

$$
\tilde{A}=\sum_{i=1}^{n} \mu_{\tilde{A}}\left(x_{i}\right) / x_{i}, x_{i} \in X
$$

and

$$
\tilde{B}=\sum_{i=1}^{m} \mu_{\tilde{B}}\left(y_{i}\right) / y_{i}, y_{i} \in X
$$

Since $\tilde{A}$ and $\tilde{B}$ are non empty fuzzy sets, then there are $n_{1}$ and $n_{2}$ positive integer numbers, such that the support of the fuzzy sets $\tilde{A}$ and $\tilde{B}$ can be given by :

$$
S(\tilde{A})=\left\{x_{1}, x_{2}, \ldots \ldots, x_{n 1}\right\} \text { and } S(\tilde{B})=\left\{x_{1}, x_{2}, \ldots \ldots, x_{n 2}\right\}
$$

Let

$$
S_{1}=S(\tilde{A}) \text { and } S_{2}=S(\tilde{B})
$$

Then define :

$$
\begin{gathered}
S_{1} \times S_{2}=\left\{\left(x, y_{j}\right) \mid x_{i} \in S_{1}, y_{j} \in S_{2}\right\}, i=1,2, \ldots \ldots n_{1}, j \\
=1,2, \ldots \ldots, n_{2}
\end{gathered}
$$

Then let

$$
I_{s_{1} \times s_{2}}=\int_{s_{1} \times s_{2}} f(u) d u=\int_{x_{i}}^{y_{j}} f(u) d u
$$

It should be noted that (8) is fuzzy set according to (2) where its membership function can be defined as:

$$
\begin{aligned}
\mu_{I s_{1} \times s_{2}}=\operatorname{Sup}_{x, y \in s_{1} \times s_{2}} \min \left\{\mu_{\tilde{A}}\left(x_{i}\right), \mu_{\tilde{B}}\left(y_{j}\right)\right\}, i \\
=1,2, \ldots \ldots, n_{1}, j=1,2, \ldots \ldots, n_{2}
\end{aligned}
$$

Let us define

$$
I_{i, j}=\int_{x_{i}}^{y_{j}} f(u) d u
$$

If $I_{i_{1}, j_{1}}=I_{i_{2}, j_{2}}, i_{1} \neq i_{2}, j_{1} \neq j_{2}$. Then the supermum of this membership function of

$I_{i_{1}, j_{1}}, I_{i_{2}, j_{2}}$ have been taken. Otherwise, no need for the supremum operation. Thus the fuzzy integration is:

$$
I(\tilde{A}, \tilde{B})=\left\{\left(I_{s_{1} \times s_{2}}, \mu\left(I_{s_{1} \times s_{2}}\right)\right) \mid I_{s_{1} \times s_{2}}=\int_{s_{1} \times s_{2}} f(u) d u\right\}
$$

Remark 6:

When $I_{i, j}=\int_{x_{i}}^{y_{j}} f(u) d u=-\int_{y_{i}}^{x_{j}} f(u) d u$, fuzzy integrals are fuzzy sets with membership function

$$
\mu_{\int_{x_{i}}^{y_{j}} f(u) d u}(u)=\mu_{\int_{y_{i}}^{x_{j}} f(u) d u}(-u)
$$

\subsection{Fuzzy Integration for Continuous Fuzzy Numbers}

For continuous membership function of the fuzzy numbers $\tilde{A}$ and $\tilde{B}$ one can develop the following.

When $\tilde{A}$ and $\tilde{B}$ are continuous fuzzy number then

$$
\begin{aligned}
& \tilde{A}=\int_{x} \mu_{\tilde{A}}(x) \backslash x, x \in X \\
& \tilde{B}=\int_{x} \mu_{\tilde{B}}(y) \backslash y, y \in X
\end{aligned}
$$

Let us define the L.R type to represent the fuzzy numbers $\tilde{A}$ and $\tilde{B}$, where the membership function are defined as follows:

$$
\mu_{\tilde{A}}(x)=\left\{\begin{array}{l}
L\left(\frac{m-x}{\alpha}\right), x \leq m \\
R\left(\frac{x-m}{\beta}\right), x \geq m
\end{array}\right.
$$

$$
\mu_{\tilde{B}}(y)=\left\{\begin{array}{l}
L\left(\frac{\dot{m}-y}{\dot{\alpha}}\right), y \leq \dot{m} \\
R\left(\frac{x-\dot{m}}{\dot{\beta}}\right), y \geq \dot{m}^{\prime}
\end{array}\right.
$$

Then

$$
\tilde{A}=(m, \alpha, \beta)_{L R}
$$

and

$$
\widetilde{B}=(\dot{m}, \dot{\alpha}, \dot{\beta})_{L R}
$$

Discretization of the above continuous fuzzy numbers $\tilde{A}$ and $\tilde{B}$ can be done in two ways, and as follows: 


\section{Discretization of a Abscissa $(x-$ axis $)$}

Let $\tilde{A}(\tilde{B})$ be a continuous fuzzy number and $m(\dot{m})$ be the mean value of the fuzzy number $\tilde{A}(\tilde{B})$ with a membership value $\mu_{\tilde{A}}(m)=1,\left(\mu_{\tilde{B}}(\dot{m})=1\right), \alpha$ and $\beta(\dot{\alpha}$ and $\hat{\beta})$ are left and right spreads of $\mu_{\tilde{A}}(x)\left(\mu_{\tilde{B}}(x)\right)$, the membership function of the continuous fuzzy number have unsharp boundaries. Furthermore, the reference function L (or R) for the fuzzy number is decreasing on ] $0,+\infty\left[\right.$, thus at $x \rightarrow \infty$, then $\mu_{\tilde{A}}(x) \rightarrow 0$

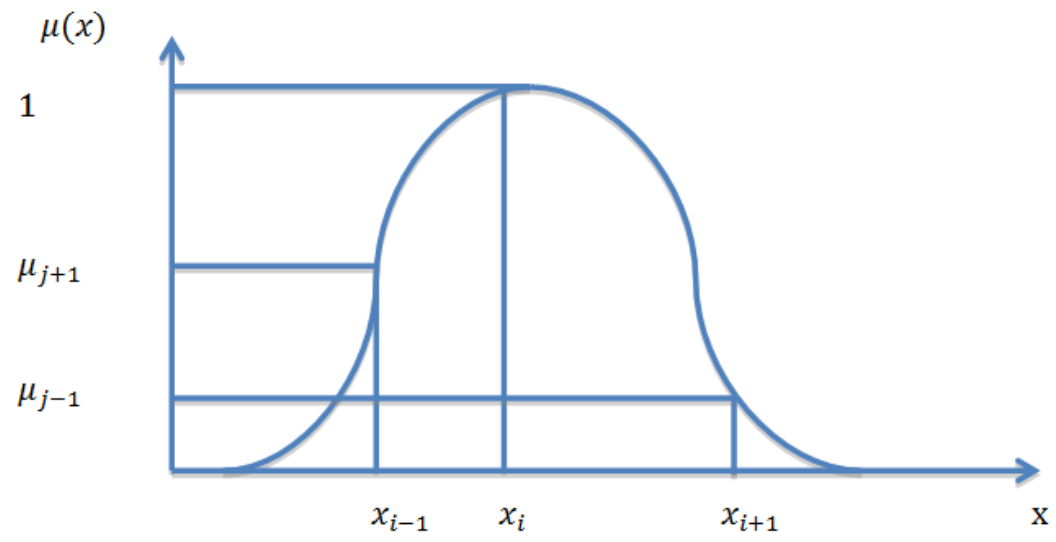

Fig. 2. Discretization of a abscissa $(x-$ axis $)$.

Step 1

Since the domain of the membership function which depending on LR-type, in general, it's not bounded, so to find the lower limit and the upper limit for the reference function $\mathrm{L}$ and $\mathrm{R}$ respectively to find the boundaries of the membership function, then we have

If $x \leq m(y \leq m)$, the membership function of the fuzzy number $\tilde{A}(\tilde{B})$ is define as

$$
\mu_{\tilde{A}}(x)=L\left(\frac{m-x}{\alpha}\right) \text { and } \mu_{B}(y)=L\left(\frac{\dot{m}-y}{\alpha}\right)
$$

Since the membership function $\mu_{\tilde{A}}(x)\left(\mu_{B}(y)\right)$ is continuous differentiable and decreasing function, therefore, there exists $p_{1} \in R\left(q_{1} \in R\right)$, such that

$$
\left|\mu_{\tilde{A}}\left(p_{1}\right)\right|<\xi_{1} \text { and }\left|\mu_{\tilde{B}}\left(q_{1}\right)\right|<\xi_{1}{ }^{*}
$$

For some small positive real number $\xi_{1}$ and $\xi_{1}{ }^{*}$, Then $p_{1}\left(q_{1}\right)$ is called the lower bounds

Similarly, if $x \geq m(y \geq m)$, the membership function of the fuzzy number $\tilde{A}(\tilde{B})$ is define as

$$
\mu_{\tilde{A}}(x)=R\left(\frac{x-m}{\beta}\right) \text { and } \mu_{B}(y)=R\left(\frac{y-m}{\hat{\beta}}\right)
$$

Since the membership function $\mu_{\tilde{A}}(x)\left(\mu_{B}(y)\right)$ is continuous differentiable and decreasing function, therefore, there exists $p_{2} \in R\left(q_{2} \in R\right)$, such that

$$
\left|\mu_{\tilde{A}}\left(p_{2}\right)\right|<\xi_{2} \text { and }\left|\mu_{\tilde{B}}\left(q_{2}\right)\right|<\xi_{2}{ }^{*}
$$

For some small positive real number $\xi_{2}$ and $\xi_{2}{ }^{*}$, Then $p_{1}$ $\left(q_{1}\right)$ is called the upper bounds

Step 2:

A partition for the continuous fuzzy number $\tilde{A}$ centered at the mean $\mathrm{v}, \mathrm{m}$ can be implemented and as follows:

$\Delta p=\frac{\left|m-p_{1}\right|}{N_{1}}($ step length for left interval, where $x \leq m)$
$\Delta p^{*}=\frac{\left|p_{2}-m\right|}{N_{2}}($ step length for left interval, where $x \geq m)$

Where

$p_{1}=$ lower limit of the membership function of $\tilde{A}$ of step 1

$p_{2}=$ upper limit of the membership function of $\tilde{A}$ of step 1

$m=$ mean value of the fuzzy number $\tilde{A}$

$N_{1}, N_{2}=$ large positive number

Similarly a partition for the continuous fuzzy number $\tilde{B}$ centered at the mean

$m$ divided into two partitions:

$\Delta q=\frac{\left|\dot{m}-q_{1}\right|}{M_{1}}($ step length for left interval, where $x \leq m)$

$\Delta p^{*}=\frac{\left|q_{2}-m^{\prime}\right|}{M_{2}}($ step length for left interval, where $x \geq m)$

Where

$q_{1}=$ lower limit of the membership function of $\tilde{B}$.

$q_{2}=$ upper limit of the membership function of $\tilde{B}$.

$\dot{m}=$ mean value of the fuzzy number $\tilde{B}$

$M_{1}, M_{2}=$ large positive number

Step 3:

Let $\quad x_{0}=m($ mean value of the fuzzy number $\tilde{A})$, then . For the left side discretized of membership function of the fuzzy number $\tilde{A}$, we have :

$$
x_{i}=x_{0}-i \Delta p, i=0,1,2, \ldots, N_{1}
$$

with a membership function

$$
\mu_{\tilde{A}}\left(x_{i}\right)=L\left(\frac{x_{0}-x_{i}}{\alpha}\right)
$$

For the right side discretized of membership function of the fuzzy number $\tilde{A}$, we have :

$$
x_{j}=x_{0}+j \Delta p^{*}, j=1,2, \ldots, N_{2}
$$

with a membership function

$$
\mu_{\tilde{A}}\left(x_{i}\right)=R\left(\frac{x_{j}-x_{0}}{\beta}\right)
$$


Thus the approximate discrete fuzzy number for continuous fuzzy number $\tilde{A}$ can be rewritten as :

$$
\begin{gathered}
\tilde{A}^{*}=\left\{\left(x_{N_{1}}, \mu\left(x_{N_{1}}\right)\right), \ldots \ldots,\left(x_{i}, \mu\left(x_{i}\right)\right), \ldots .,\left(x_{0}, 1\right),\right. \\
\left.\left(x_{1}, \mu\left(x_{1}\right)\right), \ldots .\left(x_{j}, \mu\left(x_{j}\right)\right), \ldots,\left(x_{N_{2}}, \mu\left(x_{N_{2}}\right)\right)\right\}
\end{gathered}
$$

Similarly, let $y_{0}=m$ (mean value of the membership function of $\tilde{B}$ ), then . For the left side of membership function of the fuzzy number $\tilde{B}$, we have :

$$
y_{i}=y_{0}-i \Delta q, i=0,1,2, \ldots, M_{1}
$$

with a membership function

$$
\mu_{\tilde{B}}\left(y_{i}\right)=L\left(\frac{y_{0}-y_{i}}{\alpha}\right)
$$

For the right side descritized of membership function of the fuzzy number $\widetilde{B}$, we have :

$$
y_{j}=y_{0}+j \Delta q^{*}, j=1,2, \ldots, M_{2}
$$

with a membership function

$$
\mu_{\tilde{B}}\left(y_{i}\right)=R\left(\frac{y_{j}-y_{0}}{\hat{\beta}}\right)
$$

the approximate discrete fuzzy number for continuous fuzzy number $\tilde{B}$ can be rewritten as:

$$
\begin{gathered}
\tilde{B}^{*}=\left\{\left(y_{M}, \mu\left(y_{M_{1}}\right)\right), \ldots .,\left(y_{j}, \mu\left(y_{j}\right)\right), \ldots .\right. \\
\left.\left(y_{0}, 1\right),\left(y_{1}, \mu\left(y_{1}\right)\right), \ldots\left(y_{i}, \mu\left(y_{i}\right)\right), \ldots .,\left(x_{y}, \mu\left(y_{M_{2}}\right)\right)\right\}
\end{gathered}
$$

step 4:

Take the support for the fuzzy number $\tilde{A}^{*}$ and $\tilde{B}^{*}$, then we have

$$
\begin{gathered}
S\left(\tilde{A}^{*}\right)=\left\{x_{0}, x_{1}, \ldots, x_{N_{1}+N_{2}}\right\} \\
S\left(\widetilde{B}^{*}\right)=\left\{y_{0}, y_{1}, \ldots, y_{M_{!}+M_{2}}\right\} \\
\text { Let } S_{1}=S\left(\tilde{A}^{*}\right), S_{2}=S\left(\tilde{B}^{*}\right) . \text { Define }: \\
S_{1} \times S_{2}=\left\{\left(x_{i}, y_{j}\right) \mid x_{i} \in S_{1}, y_{j} \in S_{2}, i=0,1, \ldots N_{1}+N_{2}, j\right. \\
\left.=0,1,2, \ldots, M_{1}+M_{2}\right\}
\end{gathered}
$$

Step5:

For each $(x, y) \in S_{1} \times S_{2}$, calculate the integration

$$
I_{S_{1} \times S_{2}}=\int_{S_{1} \times S_{2}} f(u) d u
$$

as has been discussed

$$
\mu_{I S_{1} \times S_{2}}=\operatorname{Sup}_{(x, y) \in S_{1} \times S_{2}} \min \left\{\mu_{\tilde{A}^{*}}, \mu_{\tilde{B}^{*}}\right\}
$$

Step 6:

Check if $I_{i_{1}, j_{1}}=I_{i_{2}, j_{2}}, i_{1} \neq i_{2}, j_{1} \neq j_{2}$, then the supremum of this membership function of $I_{i_{1}, j_{1}}, I_{i_{2}, j_{2}}$ have been take

Step 7:

Then the total fuzzy integration is :

$$
I(\tilde{A}, \tilde{B})=\left\{\left(I_{i, j}, \mu\left(I_{i, j}\right) \mid i=0,1, \ldots, K_{1}, j=0,1, \ldots, K_{2}\right\}\right.
$$

for some positive integers $K_{1}$ and $K_{2}$.

Algorithm 1. Discritization of continuous integral on (xAxis)

The following algorithm describes the necessary steps for evaluating the fuzzy interval if integral

1- For the fuzzy number $\tilde{A}$ of equation (1), compute $P_{1}$ (lower limit of the left hand side of the membership function

$L\left(\frac{m-x}{\alpha}\right)$, and $P_{2}$ (upper limit of the right hand of the membership function $R\left(\frac{x-m}{\beta}\right)$, using numerical technique to fined.

2- Let

$$
\Delta p=\frac{\left|x_{0}-p_{1}\right|}{N_{1}} \text {, and } \Delta p^{*}=\frac{\left|p_{2}-x_{0}\right|}{N_{2}}
$$

Where $\quad x_{0}=m$ (mean value $)$, and $N_{1}$ and $N_{2} \quad$ are sufficient large positive integer number

3- Compute $x_{-i}=x_{0}-i \Delta P, i=0,1,2, \ldots, N_{1}$

4- and $\mu_{\tilde{A}}\left(x_{i}\right)=L\left(\frac{x_{0}-x_{i}}{\alpha}\right)$

5- Compute $\quad x_{i}=x_{0}+\left(i-N_{1}\right) \Delta p^{*}, i=N_{1}+1, N_{1}+$ $2, \ldots, N_{1}+N_{2}$

and

$$
\mu_{\tilde{A}}\left(x_{i}\right)=R\left(\frac{x_{i}-x_{0}}{\beta}\right)
$$

6- In order to step 3 and 4 in a vector, one can set the following

$$
\begin{gathered}
x_{j}=w_{n_{1+j}}, j=-N_{1},-\left(N_{2}-1\right), \ldots, 0,1,,,, N_{2} \\
\mu_{\tilde{A}}\left(w_{j}\right)=L\left(\frac{x_{0}-w_{j}}{\alpha}\right), \mathrm{j}=0,1,2, \ldots, N_{1} \\
\mu_{\tilde{A}}\left(w_{j}\right)=R\left(\frac{w_{j}-x_{0}}{\beta}\right), j=N_{1}+1, N_{1}+2, \ldots, N_{1}+N_{2}
\end{gathered}
$$

we obtain

$$
\tilde{A}=\left\{\left(w_{j}, \mu\left(w_{j}\right) \mid j=0,1, \ldots, N_{1}+N_{2}\right\}\right.
$$

7- Repeat steps 1 - 5 for the fuzzy number $\tilde{B}$ in equation (2.1), to obtain

$$
\begin{gathered}
y_{j}=\bar{w}_{m_{1+j}}, j=-M_{1},-\left(M_{1}-1\right), \ldots, 0,1, \ldots \ldots, M_{2} \\
\mu_{\tilde{A}}\left(\bar{w}_{j}\right)=L\left(\frac{y_{0}-\bar{w}_{j}}{\alpha}\right), \mathrm{j}=0,1,2, \ldots, M_{1} \\
\mu_{\tilde{A}}\left(\bar{w}_{j}\right)=R\left(\frac{\overline{w_{J}}-y_{0}}{\beta}\right), j=M_{1}+1, M_{1}+2, \ldots, M_{1}+M_{2}
\end{gathered}
$$

We obtain

$$
\tilde{B}=\left\{\left(\overline{w_{\jmath}}, \mu\left(\overline{w_{J}}\right) \mid j=0,1, \ldots, M_{1}+M_{2}\right\}\right.
$$

8- Let the support of fuzzy number $\tilde{A}$ and $\tilde{B}$ are :

$$
S_{1}=S(\tilde{A})=\left\{w_{0}, w_{1}, \ldots, w_{N_{1}+N_{2}}\right\}
$$




$$
S_{2}=S(\tilde{B})=\left\{\bar{w}_{0},{\overline{w_{1}}}_{1}, \ldots, \bar{w}_{M+M_{2}}\right\}
$$

9- Compute

$$
I_{i, j}=\int_{w i}^{\bar{w} i} f(u) d u, \forall w_{i} \in S_{1}(\tilde{A}) \text { and } \bar{w}_{j} \in S_{2}(\tilde{B})
$$

and

$$
\mu\left(I_{i, j}\right)=\min \left\{\left(\mu_{\tilde{A}}\left(w_{i}\right), \mu_{\tilde{B}}\left(\overline{w_{J}}\right)\right\}\right.
$$

10- Sort the value of $I_{i, j}$ calculated in step 9 ascending with respect to each $I_{i, j}$ has its membership function $\mu\left(I_{i, j}\right)$

11 - If if $I_{i_{1}, j_{1}}=I_{i_{2}, j_{2}}, i_{1} \neq i_{2}, j_{1} \neq j_{2}$ then $\mu\left(I_{i, j}\right)=$ $\operatorname{Sup}\left\{\left(\mu\left(I_{i 1, j 1}\right), \mu\left(I_{i 2, j 2}\right)\right\}\right.$

12- Generate

$$
\begin{aligned}
& I(\tilde{A}, \tilde{B})=\left\{\left(I_{i, j}, \mu\left(I_{i, j}\right) \mid i=0,1,2, \ldots, k_{1}, j=\right.\right. \\
& 0,1, \ldots . ., k_{2}
\end{aligned}
$$

for positive integers $k_{1}$ and $k_{2}$ which are depending on the problem.

Using the computational above to evaluate the numerical fuzzy integration.

\section{Solution of Fuzzy Nonlinear Integral Equations}

Our treatment of Crisp nonlinear volterra ntegral equation centerel mainly on illustrations of the known methods of finding exact, or numerical solution. In this paper we present new techniques for solving crisp nonlinear volterra integral equations over the fuzzy interval .

Trapezoidal quadrature method

This approach solves a crisp nonlinear volterra integral equation of the second kind over fuzzy interval and it starts by substituting a zeroth approximation $u_{0}(x)$ in the integral equation we obtain a first approximation $u_{1}(x)$, and we will calculate the evaluate for the $u_{1}(x)$.

$$
\begin{gathered}
u(x)=f(x)+\int_{\tilde{A}}^{\tilde{B}} k\left(x, t, u(t) d t=u(x)=f(x)+I_{\tilde{A}}^{\tilde{B}}\right. \\
I_{\tilde{A}}^{\tilde{B}}=\int_{\tilde{A}}^{\tilde{B}} k(x, t, u(t) d t
\end{gathered}
$$

now we approximate the right hand integral with the repeated trapezoidal technique, then we get.

$$
\begin{aligned}
& u(x)=f(x)+\frac{h}{2} k\left(x, t_{0}\right) F\left(u_{0}\right)+h \sum_{j=1}^{n-1} k\left(x, t_{j}\right) F\left(u_{j}\right)+\frac{h}{2} k\left(x, t_{n}\right) F\left(u_{n}\right) \\
& +\frac{h^{2}}{12}\left[J\left(x, t_{0}\right) F\left(u_{0}\right)+k\left(x, t_{0}\right) u^{\prime}{ }_{0} F^{\prime}\left(u_{0}\right)-J\left(x, t_{n}\right) u_{n}-k\left(x, t_{n}\right) u_{n}^{\prime} F^{\prime}\left(u_{n}\right)\right]
\end{aligned}
$$

where $J(x, t)=\frac{\partial k(x, t)}{\partial t}$

Hence, for $x=x_{0}, x_{1}, x_{2}, \ldots \ldots, x_{n}$ in (9) we have :

This is a nonlinear system of equation and by solving it, we obtain the unknowns $u_{i}$ for $i=0,1, \ldots, n$. Then, with trapezoidal rule we can approximate the solution . by substituting $x=x_{i}$ in equation (10) we have

$$
\begin{aligned}
& u\left(x_{i}\right)=f\left(x_{i}\right)+\frac{h}{2} k\left(x_{i}, t_{0}, u\left(t_{0}\right)\right)+h \sum_{j=1}^{n-1} k\left(x_{i}, t_{j}, u\left(t_{j}\right)\right)+\frac{h}{2} k\left(x_{i}, t_{n}, u\left(t_{n}\right)\right) \\
& \quad+\frac{h^{2}}{12}\left[J\left(x_{i}, t_{0}, u\left(t_{0}\right)\right)+k\left(x_{i}, t_{0}, u\left(t_{0}\right)\right) u^{\prime}\left(t_{0}\right)-J\left(x_{i}, t_{n}, u\left(t_{n}\right)-k\left(x_{i}, t_{n}, u^{\prime}\left(t_{n}\right)\right) u^{\prime}\left(x_{n}\right)\right]\right.
\end{aligned}
$$

for $\mathrm{i}=0,1, \ldots, \mathrm{n}$. This is a system of $(\mathrm{n}+1)$ equation and $(\mathrm{n}+3)$ unknowns. By taking derivative from the equation (9), and setting $H(x, t)=\frac{\partial k(x, t)}{\partial x}$, we obtain:

$$
u^{\prime}(x)=f^{\prime}(x)+\int_{\tilde{a}}^{\tilde{b}} H(x, t, u(t)) d t
$$

Note that if $\mathrm{u}$ is a solution of equation (9), then it is also a solution of (12). By using repeated trapezoidal quadrature for (12), and placing $x=x_{i}$ we get:

$$
u^{\prime}\left(x_{i}\right)=f^{\prime}\left(x_{i}\right)+\frac{h}{2} H\left(x_{i}, t_{0}, u\left(t_{0}\right)\right)+h \sum_{j=1}^{n-1} H\left(x_{i}, t_{j}, u\left(t_{j}\right)\right)+\frac{h}{2} H\left(x_{i}, t_{n}, u\left(t_{n}\right)\right)
$$

for $\mathrm{i}=0,1, \ldots, \mathrm{n}-1$. Note that in the cases $\mathrm{i}=0, \mathrm{n}-1$, from system (13), we obtain two equations. These equations with system (11), make the nonlinear system of equation. Before we clarify further, we defined the following notation for simplicity:

$$
\begin{gathered}
f_{i}=f\left(x_{i}\right), f^{\prime}{ }_{i}=f^{\prime}{ }_{i}\left(x_{i}\right), u_{i}=u\left(x_{i}\right), u_{i}^{\prime}=u^{\prime}\left(x_{i}\right), k_{i j}=\left(x_{i}, t_{j}\right) \\
H_{i j}=H\left(x_{i}, t_{j}\right), J_{i j}=J\left(x_{i}, t_{j}\right)
\end{gathered}
$$

And

$$
u_{0}=f_{0}
$$




$$
\begin{gathered}
u_{i}=f_{i}+\sum_{i=0}^{n-1}\left(\frac{h}{2} k_{i 0}+\frac{h^{12}}{12} J_{i 0}\right) u^{2}{ }_{0}+h\left[\sum_{i=0}^{n-1} \sum_{j=1}^{n-1} k_{i j} u_{j}^{2}\right] \\
+\sum_{i=0}^{n-1}\left(\frac{h}{2} k_{i n-1}-\frac{h^{2}}{12} J_{i n-1}\right)(n-1) u^{2}{ }_{n-1}+\frac{h^{2}}{12} \sum_{i=0}^{n-1}\left(k_{i 0} u^{\prime}{ }_{0}\left(u^{2}{ }_{0}\right)^{\prime}-k_{i n-1} u_{n-1}^{\prime}\left(u^{2}{ }_{n-1}\right)^{\prime}\right) \\
u_{0}^{\prime}=f^{\prime}{ }_{0}+\frac{h}{2} H_{00}\left(u_{0}\right)^{2}+h \sum_{j=1}^{n-1} H_{0 j} u^{2}{ }_{j}+\frac{h}{2} H_{0 n-1} u^{2}{ }_{n-1} \\
u_{n-1}^{\prime}=f^{\prime}{ }_{n-1}+\frac{h}{2} H_{n-10}\left(u_{0}\right)^{2}+h \sum_{j=1}^{n-1} H_{n-1 j} u^{2}{ }_{j}+\frac{h}{2} H_{n-1 n-1} u^{2}{ }_{n-1}
\end{gathered}
$$

$\mathrm{i}=1, \ldots \ldots, \mathrm{n}-1$

By solving this system with $(n+3)$ nonlinear equations and $(n+3)$ unknowns, the approximate solution. Now we will determine the equation (1) by using LR-type representation for fuzzy are continuous functions $k(x, t)$ and $f(x)$ are continuous functions for $0 \leq x \leq$ $a$ and $0 \leq t \leq x$, the we will determine the value of fuzzy interval.

Example 1

Consider the following crisp nonlinear integral equation over the fuzzy interval

$(\tilde{A}, \tilde{B})$ be defined as

$$
u(x)=x+\int_{\tilde{0}}^{\tilde{x}}(x-t) u(t)^{2} d t
$$

the exact solution of $\operatorname{Eq}(15) \mathrm{u}(\mathrm{x})=\mathrm{x}$,

Suppose the $\tilde{x}=\tilde{1}$

By using the trapezoidal quadrature method

$$
\begin{gathered}
u_{0}(x)=x \\
u(x)=x+\int_{\tilde{0}}^{\tilde{x}}(x-t) u_{0}(t)^{2} d t
\end{gathered}
$$

Now we will solve the Eq (16) by using LR-type representation for fuzzy interval .

\section{$\tilde{A}=$ Zero fuzzy number $\tilde{0}$}

$\tilde{B}=\mathrm{x}$ fuzzy number $\tilde{x}$

now we suppose the upper limit of integral is equal $\tilde{1}$ (that mean $\tilde{x}=\tilde{1}$ ), we will use the algorithm 1 and calculate the value of the Discretization of continuous integral on $\mathrm{x}$-axis step 1 . Let $\tilde{A}$ be continuous fuzzy number of the LR-type function and as follows, where

$$
\begin{gathered}
L(x)=u_{0}(x)^{2}=f(x)=x^{2}, \\
R(x)=x
\end{gathered}
$$

Given the spread number $\alpha=2, \beta=3$ and $\mathrm{a}=\mathrm{b}=0$ (mean value of the zero fuzzy number ). Then the membership function of he continuous zero fuzzy number can be represented as follows.

$$
\mu_{\widetilde{0}}(x)=\left\{\begin{array}{c}
L\left(\frac{0-x}{2}\right)^{2}, x \in[-2,0] \\
1 x=2 \\
R\left(\frac{x-0}{3}\right) x \in[0,3]
\end{array}\right.
$$

now we calculate, $\tilde{B}$ be a continuous fuzzy number of the LR-type function as follows:

$$
L(y)=y^{2}
$$

$$
R(y)=y
$$

Given the spread number $\alpha^{\prime}=2$ and $\beta^{\prime}=3$ and $a=b=1$ (mean value of the one fuzzy number )

$$
\mu_{\widetilde{1}}(y)=\left\{\begin{array}{c}
L\left(\frac{1-y}{2}\right)^{2}, y \in[-1,1] \\
1 y=1 \\
R\left(\frac{y-1}{3}\right) y \in[1,4]
\end{array}\right.
$$

Thus, the membership functions of the continuous zero fuzzy number $\mu_{\widetilde{0}}(x)$ is bounded, where

$$
\begin{gathered}
p_{1}-2, p_{2}=3 \\
q_{1}=-1, q_{2}=4
\end{gathered}
$$

step 2. A partition for the continuous zero fuzzy number can be as follows let $x_{0}=0$ (mean value of zero fuzzy number ). For the left hand side, where $x_{0} \geq x$, let $N_{1}=4$, then

$$
\Delta p=\frac{\left|x_{0}-p_{1}\right|}{N_{1}}=0.5
$$

Also, for the right hand side, where $x_{0} \leq x$, let $N_{2}=6$

$$
\Delta p^{*}=\frac{\left|p_{2}-x_{0}\right|}{N_{1}}=0.5
$$

so, for the left side

$$
x_{i}=x_{0}-i \Delta p, i=0,1,2,3,4
$$

with the membership function

$$
\mu\left(x_{i}\right)=L\left(\frac{x_{0}-x_{i}}{2}\right)=\left(\frac{x_{i}}{2}\right)^{2}
$$

where $x_{0}=0, \alpha=2$ and $L=x^{2}$. The following results of table 1 are obtained:

Table 1. the value of the right hand side for the fuzzy lower bounded.

\begin{tabular}{lllll}
\hline $\mathbf{i}$ & & $\boldsymbol{x}_{\boldsymbol{i}}$ & & $\boldsymbol{\mu}\left(\boldsymbol{x}_{\boldsymbol{i}}\right)$ \\
\hline 0 & 0 & & 0 & \\
1 & -0.5 & 0.0625 & \\
2 & -1 & 0.25 & \\
3 & -1.5 & 0.5625 & \\
4 & -2 & 1 & \\
\hline
\end{tabular}

The right hand side

$$
x_{i}=x_{0}+i \Delta p^{*}, i=1,2,3,4,5,6
$$

with the membership function

$$
\mu\left(x_{i}\right)=R\left(\frac{x_{i}-x_{0}}{3}\right)=\left(\frac{x_{i}}{3}\right)
$$


where $x_{0}=0, \beta=2$ and $R=x$. The following results of table 2 are obtained:

Table 2. the value of the left hand side for the fuzzy lower bounded.

\begin{tabular}{lllll}
\hline $\mathbf{i}$ & & $\boldsymbol{x}_{\boldsymbol{i}}$ & & $\boldsymbol{\mu}\left(\boldsymbol{x}_{\boldsymbol{i}}\right)$ \\
\hline 1 & 0.5 & & 0.167 & \\
2 & 1 & 0.333 & \\
3 & 1.5 & 0.5 & \\
4 & 2 & 0.667 & \\
5 & 2.5 & 0.833 & \\
6 & 3 & 1 & \\
\hline
\end{tabular}

Then, the approximate discrete fuzzy number for $\tilde{A}$ is

$$
\tilde{A}^{*}=\left\{\left(x_{i}, \mu\left(x_{i}\right)\right) \mid \mathrm{i}=0,1, \ldots, 10\right\}
$$

$$
=\{(-2,1),(-1.5,0.5625),(-1,0.25),(-0.5,0.0625),(0,0),
$$

$$
(0.5,0.167),(1,0.333),(1.5,0.5),(2,0.667),(2.5,0.833),(3,1)\}
$$

The graph of continuous fuzzy number $\tilde{A}(\tilde{0})$ and it's discretization $\tilde{A}^{*}$ are shown in the following Fig 3.

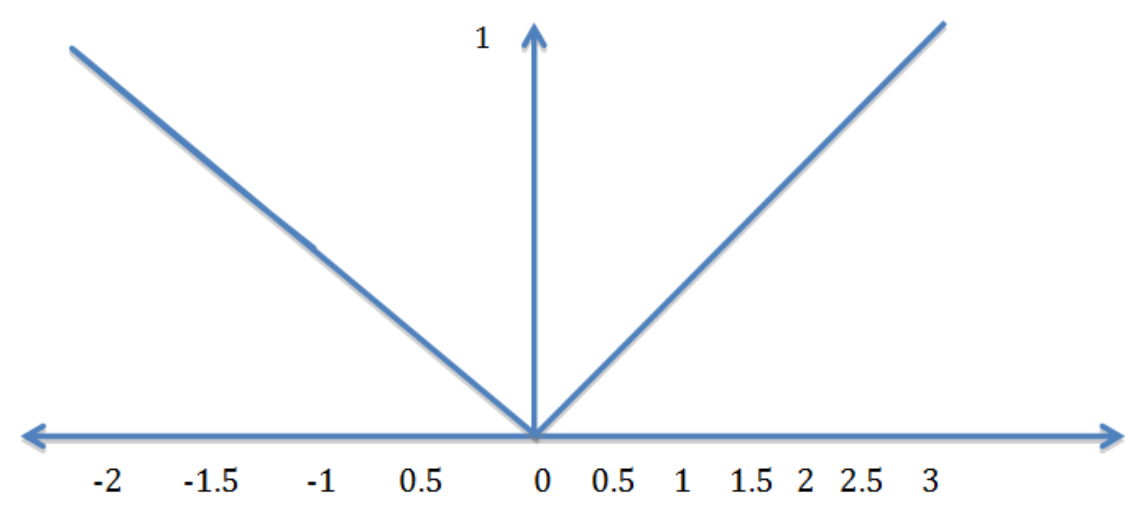

Fig. 3. Discretization of a continuous fuzzy number $\tilde{A}$

Similarly, a partition for the continuous one fuzzy number can be as follows $y_{0}=1$ (mean value of one fuzzy number). For the left hand side, where

where $y_{0} \geq y$, let $M_{1}=4$, then

$$
\Delta q=\frac{\left|y_{0}-q_{1}\right|}{M_{1}}=0.5
$$

Also, for the right hand side, where $y_{0} \leq y$, let $M_{2}=9$

$$
\Delta q^{*}=\frac{\left|p_{2}-y_{0}\right|}{M_{1}}=0.3
$$

so, for the left side

$$
y_{i}=y_{0}-i \Delta q, i=0,1,2,3,4
$$

with the membership function

$$
\mu\left(y_{i}\right)=L\left(\frac{y_{0}-y_{i}}{2}\right)=\left(\frac{y_{i}}{2}\right)^{2}
$$

where $y_{0}=1, \alpha=2$ and $L=y^{2}$. The following results of table 3 are obtained:

Table 3. the value of the right hand side for the fuzzy upper bounded.

\begin{tabular}{lllll}
\hline $\mathbf{i}$ & & $\boldsymbol{y}_{\boldsymbol{i}}$ & $\boldsymbol{\mu}\left(\boldsymbol{y}_{\boldsymbol{i}}\right)$ \\
\hline 0 & 1 & & 0 & \\
1 & 0.5 & & 0.0625 & \\
2 & 0 & 0.25 & \\
3 & -0.5 & 0.5625 & \\
4 & -1 & 1 & \\
\hline
\end{tabular}

The right hand side

$$
y_{i}=y_{0}+i \Delta q^{*}, i=1,2,3,4,5,6
$$

with the membership function

$$
\mu\left(y_{i}\right)=R\left(\frac{y_{i}-y_{0}}{3}\right)=\left(\frac{y_{i}}{3}\right)
$$

where $y_{0}=1, \beta=3$ and $R=y$. The following results of table 4 are obtained:

Table 4. the value of the left hand side for the fuzzy upper bounded.

\begin{tabular}{lllll}
\hline $\mathbf{i}$ & & $\boldsymbol{y}_{\boldsymbol{i}}$ & & $\boldsymbol{\mu}\left(\boldsymbol{y}_{\boldsymbol{i}}\right)$ \\
\hline 1 & 1.3 & & 0.1 & \\
2 & 1.6 & & 0.2 & \\
3 & 1.9 & 0.3 & \\
4 & 2.2 & 0.4 & \\
5 & 2.5 & 0.5 & \\
6 & 2.8 & 0.6 & \\
\hline
\end{tabular}

$$
\widetilde{B}^{*}=\left\{\left(y_{i}, \mu_{\tilde{B}}\left(y_{i}\right) \mid i=0,1, \ldots ., 10\right\}\right.
$$

$=\{(-1,1),(-0.5,0.5625),(0,0.25),(1,0),(1.3,0.1),(1.6,0.2)$, $(1.9,0.3),(2.2,0.4),(2.5,0.5),(2.8,0.6)\}$

The graph of continuous fuzzy number $\tilde{B}(\tilde{1})$ and it's discretization $\tilde{B}^{*}$ are shown in the following Fig 4 .

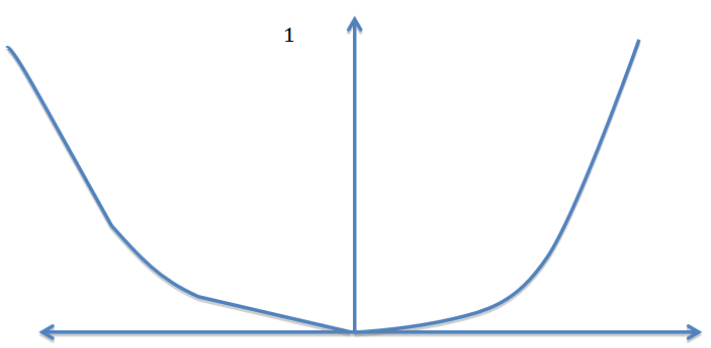

Fig. 4. Discretization of a continuous fuzzy number $\tilde{B}$. 
Step 2. Define approximately

$$
\tilde{A} \times \tilde{B} \cong\left\{\left(x_{i}, y_{j}\right) \mid x_{i} \in \tilde{A}^{*}, y_{j} \in \tilde{B}^{*}, i=0,1, \ldots . .10, j=0,1, \ldots, 10\right\}
$$

And then evaluate the integration on $\tilde{A} \times \tilde{B}$, where .

$$
\begin{gathered}
I_{i j}=\int_{x_{i}}^{y_{j}}\left(x_{i}-y_{j}\right) u_{k}\left(y_{j}\right)^{2} d y \\
\mu\left(I_{i j}\right)=\operatorname{Min}\left\{\left(\mu_{\tilde{A}}\left(x_{i}\right), \mu_{\tilde{B}}\left(y_{j}\right)\right\}\right.
\end{gathered}
$$

And now we complete the trapezoidal quadrature method

$$
\begin{gathered}
u_{0}=f_{0} \\
u_{i}=f_{i}+\sum_{i=0}^{n-1}\left(\frac{h}{2} k_{i 0}+\frac{h^{12}}{12} J_{i 0}\right) u^{2}{ }_{0}+h\left[\sum_{i=0}^{n-1} \sum_{j=1}^{n-1} k_{i j} u_{j}^{2}\right] \\
+\sum_{i=0}^{n-1}\left(\frac{h}{2} k_{i n-1}-\frac{h^{2}}{12} J_{i n-1}\right)(n-1) u_{n-1}^{2}+\frac{h^{2}}{12} \sum_{i=0}^{n-1}\left(k_{i 0} u_{0}^{\prime}\left(u^{2}{ }_{0}\right)^{\prime}-k_{i n-1} u_{n-1}^{\prime}\left(u_{n-1}{ }_{n-1}\right)\right. \\
u_{0}^{\prime}=f^{\prime}{ }_{0}+\frac{h}{2} H_{00}\left(u_{0}\right)^{2}+h \sum_{j=1}^{n-1} H_{0 j} u_{j}^{2}+\frac{h}{2} H_{0 n-1} u_{n-1}^{2} \\
u_{n-1}^{\prime}=f_{n-1}^{\prime}+\frac{h}{2} H_{n-10}\left(u_{0}\right)^{2}+h \sum_{j=1}^{n-1} H_{n-1 j} u_{j}{ }_{j}+\frac{h}{2} H_{n-1 n-1} u_{n-1}^{2}
\end{gathered}
$$

$\mathrm{n}=11$ and $\mathrm{I}=\mathrm{o}, 1, \ldots, \mathrm{n}-1$ we have 121 values of the the equation with the Minimum membership function and finally we find the $u_{i}(x)$ we have 121 values of the equation with membership function .

$[\tilde{A}, \tilde{B}]=\{[-2,1],[-2,0.5],[-2,0],[-2,-0.5],[[-2,-1],[-2,1.3],[-2,1.6],[-2,1.9],[-2,2.2],[-2,2.5],[-2,3.8],[-1.5,1],[-1.5,0.5],[-1.5,0],[-$ $1.5,-0.5],[-1.5,-1],[-1.5,1.3],[-2,1.6],[-1.5,1.9],[-1.5,2.2],[-1.5,2.5],[-1.5,3.8],[-1,1],[-1,0.5],[-1,0],[-1,-0.5],[[-1,-1],[-1,1.3],[-$ $1,1.6],[-1,1.9],[-1,2.2],[-1.5],[-1,3.8],[-0.5,1],[-0.5,0.5],[-0.5,0],[-0.5,-0.5],[[-0.5,-1],[-0.5,1.3],[-0.5,1.6],[-1.5,1.9],[-0.5,2.2],[-$ $0.5,2.5],[-0.5,3.8],[0,1],[0,0.5],[0,0],[0,-0.5],[0,1],[0,1.3],[0,1.6],[0,1.9],[0,2.2],[0,2.5],[0,3.8],[0.5,1],[0.5,0.5],[0.5,0],[0.5,-$ $0.5],[0.5,-1],[0.5,1.3],[0,1.6],[0.5,1.9],[0.5,2.2],[0.5,2.5],[0.5,3.8],[1,1],[1,0.5],[1,0],[1,-0.5],[1,-$

$1],[1,1.3],[1,1.6],[1,1.9],[1,2.2],[1,2.5],[1,3.8],[1.5,1],[1.5,0.5],[1.5,0],[1.5,-0.5],[1.5,-1],[1.5,1.3],[1.5,1.6]$,

$[1.5,1.9],[1.5,2.2],[1.5,2.5],[1.5,3.8],[2,1],[2,0.5],[2,0],[2,-0.5],[2,-1],[2,1.3],[2,1.6],[2,1.9],[2,2.2],[2,2.5],[2,3.8]$,

$[2.5,1],[2.5,0.5],[2.5,0],[2.5,-0.5],[2.5,-1],[2.5,1.3],[2.5,1.6],[2.5,1.9],[2.5,2.2],[2.5,2.5],[2.5,3.8],[3,1],[3,0.5],[3,0],[3,-$

$$
0.5],[[3,-1],[3,1.3],[3,1.6],[3,1.9],[3,2.2],[3,2.5],[3,3.8]\}=[-\tilde{1}, \tilde{4}]
$$

And the value of membership function of interval of crisp integration is for all the interval above as follows:

$$
\mu[\widetilde{A}, \widetilde{B}]=\operatorname{Min}\{0,0,0,0,0,0,0,0,0,0,0,0,0.0625,0625,0625,0625,0625,0625,0625,0625,
$$

$0625,0625,0,0625,0,0.25,0.25,0.25,0.167,0.25,0.25,0.25,0.25,0.25,0.250,0.625,0,25,0.5625,0.5625,0.167,0.333,0.5,0.5625,0.56$ $25,0.5625,0,0,0.625,0.29,0.5625,1,0.167,0.333,0.5,0.667,0831,1,0,0.0625,0.1,0.1,0.1,0.0 .1,0.1,0.1,0.1,0.1,0.1,0,0.0625,0.2,0.2$, $0.2,0.2,0.2,0.2,0.2,0.2,0.2,0,0.0625,0.25,0.3,0.3,0.3,0.3,0.3,0.3,0.3,0.3,0,0.0625,0.25,0.4,0.4,0.167,0.333,0.4,0.4,0.4,0.4,0,0.062$ $5,0.25,0.5,0.5,0.267,0.333,0.5,0.5,0.5,0.5,0,0.0625,0.250 .5265,0.6,0.167,0.333,0.5,0.6,0.6,0.6\}$

step 3. Check if $I_{i 1, j 1}=I_{12, j 2}, i 1 \neq j 2, i 2 \neq j 2$, then

$$
\mu\left(I_{i, j}\right)=\operatorname{Sup}\left\{\mu\left(I_{i 1, j 1}\right), \mu\left(I_{12, j 2}\right)\right\}
$$

we have the number of crisp nonlinear function over fuzzy integration are 121 values with membership function . step 4. Finally, we have the total crisp nonlinear function over fuzzy integration

$$
\left.I(\tilde{A}, \tilde{B})=I(\tilde{0}, \tilde{1})=\left\{\left(I_{i j}, \mu\left(I_{i, j}\right)\right)\right) \mid i=0,1, \ldots, n-1 \in N ; j=0,1, \ldots, J \in n-1\right\}
$$

Now we will take only one interval to find the value of crisp integral $u_{i}$ over the fuzzy interval, and we will using the system of nonlinear integral equation by using trapezoidal technique and comparing with the exact solution and calculate the absolute error. Table 1

We will take the first interval $[-2,1]$, let partition for this interval 
Let $n=5, h=\frac{b-a}{n}=\frac{1-(-2)}{5}=0.6$ we will added 0.6 for all step between $[-2,1]$

Table 5. Compares between the exact solution and quadrature method with absolute error.

\begin{tabular}{llll}
\hline $\mathbf{x}$ & $\mathbf{E x a c t}=\mathbf{u}(\mathbf{x})=\mathbf{x}$ & $\begin{array}{l}\text { Trapezoidal } \\
\text { technique }\end{array}$ & Absolute error \\
\hline-2 & -2 & -1.942536 & 0.074641 \\
-1.4 & -1.4 & -1.357654 & 0.042346 \\
-0.8 & -0.8 & -0.763452 & 0.036548 \\
-0.2 & -0.2 & -0.183245 & 0.016755 \\
0.2 & 0.2 & 0.197621 & 0.002379 \\
0.6 & 0.6 & 0.558754 & 0.041746 \\
1 & 1 & 0.998001 & 0.001499 \\
\hline
\end{tabular}

The table 5 discuses between the numerical method and exact solution is successes to find the value of the crisp nonlinear integral equation over fuzzy interval with a small absolute error . Similarly we can find the all interval above when $n=5$ that mean we will have 605 results for all interval and if $n=10$ that mean we will have 1210 value .

\section{Conclusion}

In this paper, a numerical method based on quadrature methods has been proposed to approximate the solution of crisp nonlinear integral equation of a second kind over fuzzy interval. In this method, the problem of solving crisp nonlinear integral equation over fuzzy interval reduced to a problem of solving a system of algebraic equation. The recommendation for future work we will defined the value of crisp nonlinear integration equation over fuzzy interval over $\mu$-axis, Illustrative examples are prepared to show the efficiency and simplicity of the method.

\section{References}

[1] K. E. Atkinson, The Numerical solution of Integral Equation of the Second Cambridge University Press, 1997.

[2] A. Alipanah and M. Dehghan, Numerical solution of the nonlinear Fredholm integral equations by positive definitions. Appl. Math. Comput., 190(2007), 1754-176.

[3] J. Store and R. Bulirsch, Introduction to Numerical Analysis, Second ed, Springer-Verlag, 1993.

[4] Chen, S. H. 1985. Operations on fuzzy numbers with function principle. Tamkang journal of Management Science, 6:13-25

[5] R. Goetschel, W. Voxman, Elementary calculate, fuzzy Sets System 18 (1986)31-43
[6] C. T. H. Baker, A perspective on the numerical treatment of volterra equations, Journal of Computational and Applied Mathematics, 125 (2000), 217-249.

[7] D. Dubois and H. Prade, Operations on fuzzy numbers, International Journal of Systems Science, 9 (1978), 613-626.

[8] A. Kaufmann and M. M. Gupta, Introduction fuzzy arithmetic, Van Nostrand Reinhold, New York, 1985

[9] S. Abbasbandy, E. Babolian and M. Alavi, Numerical method for solving linear fredholm fuzzy integral equations of the second kind, Chaos Solitons\& Fractals, 31 (2007), 138-146.

[10] T. Allahviranloo and M. Otadi, Gaussian quadratures for approximate of fuzzy multiple integrals, Applied Mathematics and Computation, 172 (2006), 175-187.

[11] M. Ma, M. Friedman and A. Kandel, A new fuzzy arithmetic, Fuzzy Sets and Systems, 108 (1999), 83-90

[12] C. T. H. Baker, A perspective on the numerical treatment of volterra equations, Journal of Computational and Applied Mathematics, 125 (2000), 217-249

[13] A. M. Bica, Error estimation in the approximation of the solution of nonlinear fuzzy fredholm integral equations, Information Sciences, 178 (2008), 1279-1292

[14] D. Dubois and H. Prade, Operations on fuzzy numbers, International Journal of Systems Science, 9 (1978), 613-626.

[15] G. J. Klir, U. S. Clair and B. Yuan, Fuzzy set theory: foundations and applications, Prentice-Hall, 1997.

[16] W. Congxin and M. Ming, On embedding problem of fuzzy number spaces, Part 1, Fuzzy Sets and Systems, 44 (1991), 33-38.

[17] M. L. Puri and D. Ralescu, Fuzzy random variables, Journal of Mathematical Analysis and Applications, 114 (1986), 409-422.

[18] [Eman A.hussain, Existence and uniqueness of the solution of nonlinear integral equation, Department of mathematics /college of science ,university of Al-mustansiriyah Iraq/ Baghdad ,vol.26(2)2013

[19] Kandel, A., "Fuzzy Mathematical Techniques with applications", Addison Wsely publishing Company, Inc., (1986)

[20] Negotia, C. V.m Ralescu, D. A., "Application of Fuzzy Sets to System Analysis "Basel, Stuttgart, (1975)

[21] Zadeh, L. A., "fuzzy Sets”, Information Control, Vol.8, (1965), pp338-353

[22] Dubois, D. and Prade, H., "Fuzzy Sets and System: Theory and Application", Academic Press, Inc., (1908). 